\title{
RAMANUJAN'S METHOD IN q-SERIES CONGRUENCES
}

\author{
BY
}

\author{
George E. Andrews ${ }^{(1)}$ And Ranjan Roy \\ Written in honor of Herb Wilf's $65^{\text {th }}$ birthday
}

\begin{abstract}
We show that the method developed by Ramanujan to prove $5 \mid p(5 n+4)$ and $7 \mid p(7 n+5)$ may, in fact, be extended to a wide variety of $q$-series and products including some with free parameters.
\end{abstract}

\section{Introduction.}

Ramanujan [11] is the discoverer of the surprising fact that the partition function, $p(n)$, satisfies numerous congruences. Among the infinite family of such congruences, the two simplest examples are

$$
p(5 n+4) \equiv 0 \quad(\bmod 5)
$$

and

$$
p(7 n+5) \equiv 0 \quad(\bmod 7)
$$

Ramanujan used an ingenious and elementary argument to prove these congruences which relied on Jacobi's famous formula 10; last eqn. p.5]:

$$
(q ; q)_{\infty}^{3}=\prod_{n=1}^{\infty}\left(1-q^{n}\right)^{3}=\sum_{j=0}^{\infty}(-1)^{j}(2 j+1) q^{j(j+1) / 2}
$$

where

$$
(A)_{N}=(A ; q)_{N}=\prod_{j=0}^{N-1}\left(1-A q^{j}\right) .
$$

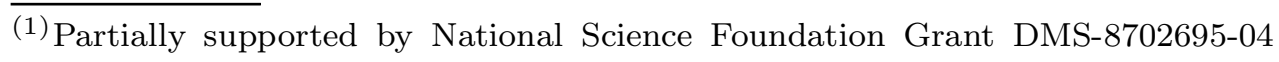


A rather more general result of this nature was proved in $[3 ;$ p. 27, Th. 10.1] to account for certain congruences connected with generalized Frobenius partitions.

Indeed J. M. Gandhi [7], [8], [9], J. Ewell [5], L. Winquist [12] and many others (cf., Gupta 10; Sec. 6.3]) have proved partition function congruences based on this idea. In all these theorems, the underlying generating functions were either modular forms or simple linear combinations thereof.

The point of this paper is to show that Ramanujan's original method is applicable to an infinite number of congruence theorems including many non-modular functions defined by $q$-series.

Our main result is:

Theorem 1. Suppose $p$ is a prime $>3$, and $0<a<p$ and $b$ are integers. Also, $-a$ must be a quadratic nonresidue mod $p$. Suppose $\left\{\alpha_{n}\right\}_{n=-\infty}^{\infty}=\left\{\alpha_{n}\left(z_{1}, z_{2}, \ldots, z_{j}\right)\right\}$ is a doubly infinite sequence of Laurent polynomials over $\mathbb{Z}$ with variables $z_{1}, \ldots, z_{j}$ independent of $q$. Then there is an integer $c$ such that the coefficient of $z_{1}^{m_{1}} z_{2}^{m_{2}} \cdots z_{j}^{m_{j}}$ $q^{p N}$ in

$$
\frac{q^{c} \sum_{n=-\infty}^{\infty} \alpha_{n} q^{a\left(\begin{array}{c}
n \\
2
\end{array}\right)+b n}}{(q ; q)_{\infty}^{p-3}}
$$

is divisible by $p$. For each integer $m$, we shall denote by $\bar{m}$ the multiplicative inverse of $m \bmod p$. The integer $c=c_{p}(a, b)$ may be chosen as the least nonnegative integer congruent to $\overline{8}\left(a(2 b \bar{a}-1)^{2}+1\right) \bmod p$.

In Section 2, we shall prove this result. In Section 3 we examine the implications of Theorem 1 for a variety of modular forms. In Section 4, we collect a number of congruences for the coefficients in several $q$-series.

\section{The Proof of Theorem 1.}

With the various hypotheses of the theorem, we note that 


$$
\begin{gathered}
\frac{q^{c} \sum_{n=-\infty}^{\infty} \alpha_{n} q^{a\left(\begin{array}{c}
n \\
2
\end{array}\right)+b n}}{(q ; q)_{\infty}^{p-3}} \\
=\frac{q^{c} \sum_{n=-\infty}^{\infty} \sum_{j=0}^{\infty}(-1)^{j}(2 j+1) \alpha_{n} q^{a\left(\begin{array}{c}
n \\
2
\end{array}\right)+b n+j(j+1) / 2}}{(q ; q)_{\infty}^{p}} \\
\equiv \frac{q^{c} \sum_{n=-\infty}^{\infty} \sum_{j=0}^{\infty}(-1)^{j}(2 j+1) \alpha_{n} q^{a\left(\begin{array}{c}
n \\
2
\end{array}\right)+b n+j(j+1) / 2}}{\left(q^{p} ; q^{p}\right)_{\infty}}(\bmod p) .
\end{gathered}
$$

We see that in this last expression the denominator is a function of $q^{p}$. Let us now examine the exponent of $q$ in the numerator; for ease of computation we multiply by 8:

$$
\begin{aligned}
& 8\left(c+a\left(\begin{array}{l}
n \\
2
\end{array}\right)+b n+j(j+1) / 2\right) \\
= & 8 c+a\left(4 n^{2}-4 n\right)+8 b n+4 j^{2}+4 j \\
\equiv & a(2 n+2 b \bar{a}-1)^{2}+(2 j+1)^{2} \quad(\bmod p)
\end{aligned}
$$

Now we observe (by the definition of $c$ ) that if $j \equiv(p-1) / 2 \bmod p$ (i.e. $(2 j+1) \equiv$ $0(\bmod p))$, then the last expression above is congruent to $0 \bmod p$ precisely when

$$
n \equiv(1-2 b \bar{a}) \overline{2} \equiv \frac{p+1}{2}-b \bar{a} \quad(\bmod p)
$$

If $j \not \equiv \frac{p-1}{2}(\bmod p)$, then the last expression in $(2.2)$ can never be congruent to zero $\bmod p$ because by the conditions on $a$

$$
-a(2 n+2 b \bar{a}-1)^{2}
$$

is either 0 or a quadratic nonresidue $\bmod p$ and so cannot be congruent to a quadratic residue (i.e. $\left.(2 j+1)^{2}\right) \bmod p$.

Hence the coefficients of $q^{p N}$ in $(2.1)$ will all be linear combinations over $p \mathbb{Z}$ of various $\alpha_{n}$ (which are Laurent polynomials in several variables over $\mathbb{Z}$ ). 


\section{Modular Forms.}

Ramanujan, Ewell, Gandhi (and probably many others) have proved instances of Theorem 1 (as mentioned in Section 1).

Congruence (1.1) follows from Theorem 1 with $p=5, a=3, b=1, c_{5}(3,1)=1$ and $\alpha_{m}=(-1)^{m}$. Congruence $(1.2)$ follows from Theorem 1 with $p=7, a=b=$ $1, c_{7}(1,1)=2$ and $\alpha_{m}=(-1)^{m}(2 m+1)$ if $m \geqq 0, \alpha_{m}=0$ if $m<0$.

Gandhi's Theorem IV in [7] corresponds to $\alpha_{m}=\delta_{m, 0}$, while Theorem 2 in [8] corresponds to $a=b=1$ and $\alpha_{m}=(-1)^{m}(2 m+1)$ if $m \geqq 0, \alpha_{m}=0$ if $m<0$. Finally, Theorem 4 in $[8]$ corresponds to $a=3, b=1$ and $\alpha_{m}=(-1)^{m}$.

Theorem 10.1 of $[3]$ is the case $p=5, a=2, b=1, c_{5}(2,1)=2$; in that result the $\alpha_{m}$ were assumed to be 0 if $m<0$ and to be integers otherwise.

The generality of Theorem 1 allows for a variety of other modular forms. To illustrate, we consider

$$
\sum_{n=0}^{\infty} V_{n} q^{n}=\frac{\sum_{n=0}^{\infty} p(n) q^{n}}{\sum_{n=0}^{\infty}(-1)^{n} r_{2}(n) q^{n}}
$$

where $r_{2}(n)$ is the number of representations of $n$ as a sum of two squares. We note that

$$
\begin{aligned}
\sum_{n=0}^{\infty} V_{n} q^{n} & =\frac{1}{(q)_{\infty}\left(\sum_{n=-\infty}^{\infty}(-1)^{n} q^{n^{2}}\right)^{2}} \\
& =\frac{(-q)_{\infty}^{2}}{(q)_{\infty}^{3}} \\
& =\frac{\left(q^{2} ; q^{2}\right)_{\infty}}{(q)_{\infty}^{4}\left(q ; q^{2}\right)_{\infty}} \\
& =\frac{\sum_{m=0}^{\infty} q^{m(m+1) / 2}}{(q)_{\infty}^{4}}
\end{aligned}
$$

Now by Theorem 1 with $p=7, a=b=1, c_{7}(1,1)=2, \alpha_{m}=1$ if $m \geq 0$, and 0 if $m<0$, we see that

$$
V_{7 m+5} \equiv 0 \quad(\bmod 7)
$$




\section{4. $q$-Series.}

Of course, our point here is not to extend slightly Ramanujan's basic idea to a few more modular forms. Rather we hope to illustrate its applicability to $q$-series.

Theorem 2. For any prime $p \equiv 3(\bmod 4)$ with $4 c \equiv 1(\bmod p)$, the coefficient of $z^{m} q^{p n-c}$ in

$$
\frac{(z q)_{\infty}}{(q)_{\infty}^{p-4}} \sum_{n=0}^{\infty} \frac{q^{n}}{(q)_{n}(z q)_{n}}
$$

is divisible by $p$.

Proof. By Heine's transformation [1; Cor. 2.3, p. 19]

$$
\sum_{n=0}^{\infty} \frac{q^{n}}{(q)_{n}(z q)_{n}}=\frac{1}{(q)_{\infty}(z q)_{\infty}} \sum_{n=0}^{\infty}(-1)^{n} q^{n(n+1) / 2} z^{n}
$$

Now apply Theorem 1 with $a=b=1$ and $c \equiv \overline{4}(\bmod p)$.

Theorem 3. For any prime $p \equiv 5$ or $7(\bmod 8)$ with $8 c \equiv 1(\bmod p)$, the coefficient of $z^{m} q^{p n-c}$ in

$$
\frac{1}{(q)_{\infty}^{p-3}} \sum_{n=0}^{\infty} \frac{(z)_{n+1} z^{n}}{(-z q)_{n}}
$$

is divisible by $p$.

Proof. By the Rogers-Fine identity 6; p. 15, eqn. (14.31)]

$$
\sum_{n=0}^{\infty} \frac{(z)_{n+1} z^{n}}{(-z q)_{n}}=1+2 \sum_{n \geq 1}\left(-z^{2}\right)^{n} q^{n^{2}}
$$

Now apply Theorem 1 with $a=2, b=1$ noting that for $p \equiv 5(\bmod 8) 2$ is a non-quadratic residue, and for $p \equiv 7(\bmod 8), 2$ is a quadratic residue. Also we must have $c \equiv \overline{8}(\bmod p)$.

Theorem 4. For any prime $p \equiv 5$ or $11(\bmod 12)$, the coefficient of $q^{p n-(p+1) / 2}$ in

$$
\frac{1}{(q)_{\infty}^{p-4}} \sum_{n=0}^{\infty}\left[\begin{array}{c}
2 n \\
n
\end{array}\right] q^{n}
$$


(where $\left[\begin{array}{l}A \\ B\end{array}\right]=(q)_{A} /\left((q)_{B}(q)_{A-B}\right)$ is the q-binomial coefficient) is divisible by $p$.

Proof. By Lemma 3 of 2; p. 159],

$$
\frac{1}{(q)_{\infty}} \sum_{n=0}^{\infty}(-1)^{n} q^{3 n(n+1) / 2}=\sum_{n=0}^{\infty}\left[\begin{array}{c}
2 n \\
n
\end{array}\right] q^{n} .
$$

Now apply Theorem 1 with $a=b=3$ noting that for $p \equiv 5(\bmod 12), 3$ is a non-quadric residue and for $p \equiv 11(\bmod 12), 3$ is a quadratic residue.

Theorem 5. For any prime $p \equiv 5$ or $11(\bmod 12)$ and $6 c \equiv 1(\bmod p)$, the coefficient of $q^{p n-c}$ in

$$
\frac{1}{(q)_{\infty}^{p-4}} \sum_{n=1}^{\infty} \frac{q^{n^{2}}}{\left(1-q^{n}\right)\left[\begin{array}{c}
2 n \\
n
\end{array}\right]}
$$

is divisible by $p$.

Proof. By (5.1) of $[4 ;$ p. 272]

$$
\begin{gathered}
\sum_{n=1}^{\infty} \frac{q^{n^{2}}}{\left(1-q^{n}\right)\left[\begin{array}{c}
2 n \\
n
\end{array}\right]} \\
=\sum_{n=1}^{\infty} \frac{q^{n^{2}}(q)_{n-1}}{\left(q^{n+1}\right)_{n}}=\frac{1}{(q)_{\infty}} \sum_{n=-\infty}^{\infty}(-1)^{n-1} n q^{n(3 n-1) / 2} .
\end{gathered}
$$

Now apply Theorem 1 with $a=3, b=1$. As in Theorem $4, p$ must be $\equiv 5$ or 11 $\bmod 12$, and now $6 c=1(\bmod p)$.

\section{Conclusion.}

There are undoubtedly significant extensions of the ideas we have presented here. We note that Winquist's proof that

$$
p(11 n+6) \equiv 0 \quad(\bmod 11)
$$

does not fit into Theorem 1. However now that the application of (1.3) to Theorem 1 has been made, it should be possible to find a variety of congruences for the coefficients of other $q$-series. In addition, the function given in Theorem 1 may be multiplied by any function $f_{1}(q) \in \mathbb{Z}[[q]]$ for which

$$
f_{1}(q) \equiv f_{2}\left(q^{p}\right) \quad(\bmod p) .
$$


Finally, it may well be asked what happens when $a$ is a quadratic residue mod $p$ and $p \equiv 1(\bmod 4)$ or $a$ is a quadratic non-residue and $p \equiv 3(\bmod 4)$. It is not difficult to show that our analysis produces indices $j \not \equiv(p-1) / 2(\bmod p)$ which make the exponent on $q$ congruent to zero $\bmod p$ irrespective of $c_{p}(a, b)$. Consequently one would have to invoke special conditions on the $\alpha_{m}$ to produce coefficients that are multiples of $p$.

\section{REFERENCES}

1. G.E. Andrews, The Theory of Partitions, Encyclopedia of Math. and Its Applications, Vol. 2, Addison-Wesley, Reading, 1976 (Reissued: Cambridge University Press, Cambridge, 1985).

2. G.E. Andrews, Ramanujan's "lost" notebook: I. partial $\theta$-functions, Adv. in Math. 41 (1981), 137-172.

3. G.E. Andrews, Generalized Frobenius partitions, Memoirs of the Amer. Math. Soc., 49(1984), No. 301, iv+, 44 pp.

4. G.E. Andrews, Bailey chains and generalized Lambert series: I. Four identities of Ramanujan, Illinois J. Math. 36 (1992), 251-274.

5. J.A. Ewell, Completion of a Gaussian derivation, Proc. Amer. Math. Soc. 84 (1982), 311-314.

6. N.J. Fine, Basic Hypergeometric Series and Applications, Math. Surveys No. 27, Amer. Math. Soc., Providence, 1988.

7. J.M. Gandhi, Congruences for $p_{r}(n)$ and Ramanujan's $\tau$ function, Amer. Math. Monthly 70 (1963), 265-274.

8. J.M. Gandhi, Generalization of Ramanujan's congruences $p(5 m+4) \equiv 0(\bmod 5)$ and $p(7 m+$ $5) \equiv 0(\bmod 7)$, Monatsch. Math. 69 (1965), 389-392.

9. J.M. Gandhi, Some congruences for $k$-line partitions of a number, Amer. Math. Monthly $\mathbf{7 4}$ (1967), 179-181.

10. H. Gupta, Partitions - a survey, Journal of Res. of Nat. Bur. Standards - B Math. Sciences 74B (1970), 1-29.

11. S. Ramanujan, Some properties of $p(n)$, the number of partitions of $n$, Proc. Cambridge Phil. Soc., 19(1919), 207-210 (Reprinted: Coll. Papers., Cambridge Univ. Press, Cambridge, 1927, Reissued: Chelsea, New York, 1962).

12. L. Winquist, An elementary proof of $p(11 m+6) \equiv 0(\bmod 11)$, J. Combinatorial Theory 6 (1969), 56-59.

The Pennsylvania State University

University Park, PA 16802

email: andrews@math.psu.edu

and

Beloit College

Beloit, Wisconsin

email: royr@beloit.edu 